\title{
Efecto del ejercicio aeróbico y la estimulación ambiental sobre la reducción de los niveles de ansiedad en el envejecimiento
}

\author{
Patricia Sampedro \\ Universidad de Oviedo (España)
}

\begin{abstract}
El enriquecimiento ambiental (EAM) y el ejercicio aeróbico (EJ) son intervenciones capaces de reducir la ansiedad durante el envejecimiento, pero poco se sabe acerca de cómo modulan las proyecciones cerebrales hacia el eje hipotálamo-pituitario adrenal (HPA). Estudiamos el efecto de un programa de EAM y de EJ durante 2 meses en ratas Wistar macho de 18 meses de edad asignadas aleatoriamente a tres grupos: control $(\mathrm{CO}, N=6)$, EAM $(N=8)$ y EJ $(N=8)$. El programa de ejercicio fue llevado a cabo $15 \mathrm{~min} /$ día y el grupo EAM fue estabulado en una jaula de grandes dimensiones con diferentes objetos renovados frecuentemente. Mediante la histoquímica de la citocromo c oxidasa (COx) estudiamos la actividad metabólica de regiones cerebrales implicadas en la respuesta de ansiedad. El EAM redujo la actividad metabólica de regiones cerebrales implicadas en activar al eje HPA (corteza infralímbica, amígdala basolateral y núcleo paravetricular hipotalámico, $(p<0.05)$. Por otro lado, el EJ aumentó la actividad de regiones implicadas en la inhibición (corteza cingulada, núcleo del lecho de la estría terminal e hipocampo dorsal, $(p<0.05)$. En conclusión, parece que el EAM y el EJ modulan de forma diferente la actividad de regiones cerebrales que proyectan al eje HPA y podrían constituir intervenciones eficaces para reducir los niveles de ansiedad durante el envejecimiento.
\end{abstract}

Palabras clave: Envejecimiento, enriquecimiento ambiental, ejercicio aeróbico, rata Wistar.

Effect of the aerobic exercise and the environmental enrichment on the reduction of the anxiety levels in the aging. The environmental enrichment (EE) and the aerobic exercise (EX) are interventions capable of reducing anxiety levels in the aging, but few is known about how they modulating the projections to the hypothalamic-pituitary axis (HPA). We studied the effect of an EE and EX programs carried out during two months in 18 month-old Wistar rats assigned to 3 groups: $(\mathrm{CO}, N=6), \mathrm{EE}(N=8)$ y $\mathrm{EX}(N=8)$. The EX program was carried out during $15 \mathrm{~min} /$ day and the EE group was housed in a big cage with different objects frequently changed. Through the cytochrome c oxidase histochemistry (COx), we analysed the metabolic activity of several brain regions involved in the anxiety response. The EE reduced the brain activity of regions involved in the activation of the HPA axis (infralimbic cortex, basolateral amygdala and the hypothalamic paraventricular nucleus $(p<0.05)$. On the other hand, the EX program increased the activity of brain regions involved in the inhibition of the HPA axis (cingulate cortex, bed nucleus of the stria terminalis and the dorsal hippocampus $(p<0.05)$. In conclusion, it seemed that the EE and the EX modulate in different way the activity of brain regions that project to the HPA axis and they could constitute successful interventions to reduce the anxiety levels in the aging.

Keywords: Aging, environmental enrichment, aerobic exercise, Wistar rat.

Correspondencia: Patricia Sampedro Piquero. Facultad de Psicología. Universidad de Oviedo. Plaza Feijoo, s/n. C.P.: 33003. Oviedo (España). E-mail: patrisampiq@ gmail.com 
En la actualidad, el cambio demográfico de nuestro país y del resto del mundo ha hecho que la investigación en el área del envejecimiento sea fundamental y de gran necesidad. Uno de los factores a los que se debe este envejecimiento de la población es el aumento de la esperanza de vida, como recientemente ha puesto de manifiesto el Instituto Nacional de Estadística (INE) así como, la caída de la fecundidad. De este modo, a mitad de siglo XXI se espera que el 37.6\% de los españoles tenga 65 años o más, lo que equivaldría a unos 16.4 millones de personas, y esta tendencia se mantendrá en los años siguientes (Christensen, Doblhammer, Rau, y Vaupel, 2009). Una de las consecuencias de este cambio demográfico es el incremento de la incidencia de patologías relacionadas con el envejecimiento, como es el caso de las enfermedades neurodegenerativas (Bishop, Lu, y Yanker, 2010; O’Callaghan, Griffin, y Kelly, 2009). Con el aumento de la edad se observa un cierto declive en algunas funciones cognitivas y aunque no es un hecho generalizado, existe un porcentaje relativamente alto de personas que experimentan déficits cognitivos, especialmente problemas de memoria (Deary et al., 2009). Así, en torno a un $20 \%$ de la población mayor sufre deterioro cognitivo leve (Petersen, 2011) y entre un 5-10\% desarrolla demencia (Plassman et al., 2008). Incluso, datos más alarmantes ofrecidos por la Organización Mundial de la Salud (OMS) y la Asociación Internacional de Alzheimer (2013) revelan que cada año aparecen 7.7 millones de nuevos casos de demencia, lo que equivale a un caso nuevo cada cuatro segundos.

Con el envejecimiento también se ha descrito un aumento de conductas ansiosas lo que hace que los trastornos de ansiedad sean muy comunes en estas edades (Wolitzky-Taylor, Castriotta, Lenze, Stanley, y Craskey, 2010). Concretamente, a medida que envejecemos existe un alterado funcionamiento del eje hipotálamo-pituitario adrenal (HPA) caracterizado por una secreción excesiva de glucocorticoides (GC) y una reducida retroalimentación negativa que le impide volver a sus niveles de actividad basal (Eichenbaum, Yonelinas, y Ranganath, 2007; Prenderville, Kennedy, Dinan, y Cryan, 2014; Tasker y Herman, 2011). Esa feedback inhibitorio es mediado a través de receptores específicos $(\mathrm{RG})$ que se encuentran en diferentes regiones cerebrales y que con el envejecimiento muestran una expresión reducida (Bizon, Lee, y Gallagher, 2001; Garrido, 2011). Algunas de las consecuencias de los elevados niveles de GC en sangre es un descenso del número de neuronas en el hipocampo junto con un aumento de su vulnerabilidad (Jöels, Karst, De Rijk, y de Kloet, 2008; Sandi y Pinelo-Nava, 2007). El hipocampo es una región cerebral tradicionalmente relacionada con los procesos de aprendizaje y memoria, siendo con el paso de los años, y sobre todo en casos de enfermedades neurodegenerativas, una de las regiones más afectadas (Fjell, McEvoy, Holland, Dale, y Walhovd, 2014). Igualmente, el hipocampo también se ha relacionado con funciones de carácter emocional al contar con niveles elevados de RG (Prickaerts y Steckler, 2005). 
Figura 1. La presencia de un estresor dispara la liberación de GC debido a la activación del eje HPA
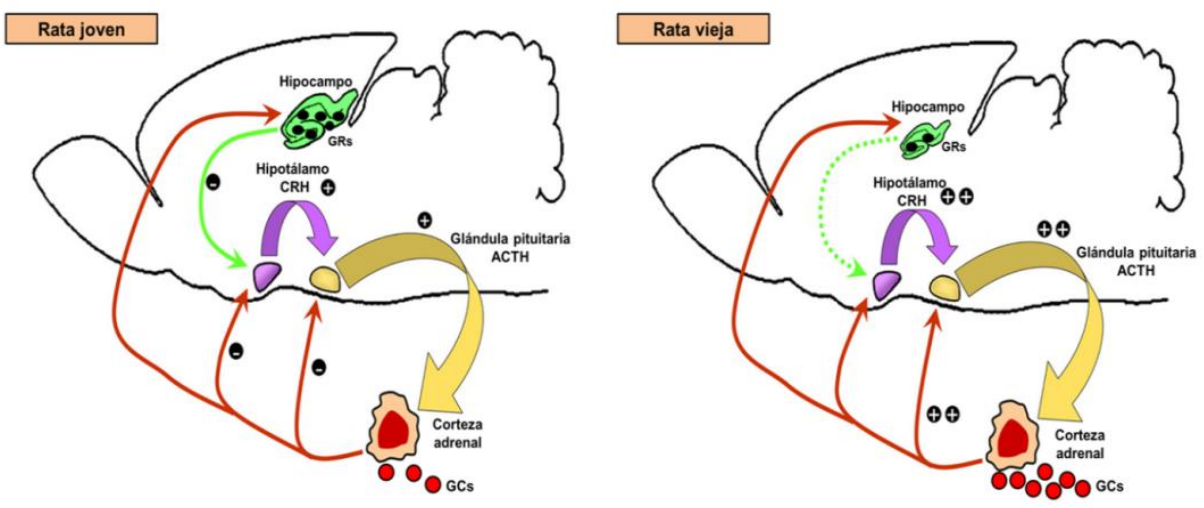

Los GC tienen la capacidad de atravesar la barrera hematoencefálica y unirse a RG distribuidos abundantemente en regiones como el hipocampo. En roedores jóvenes, el hipocampo es capaz de ejercer un preciso control inhibitorio de la actividad del eje HPA permitiendo volver a niveles basales de activación, mientras que en roedores viejos existe una falta de control inhibitorio sobre la actividad de este eje.

De este modo, las perspectivas actuales consideran que los déficits de memoria declarativa que aparecen durante el envejecimiento no son debidos exclusivamente a las alteraciones anatómicas o neuroquímicas en las regiones cerebrales implicadas en esta función cognitiva, sino que cambios en sistemas moduladores, como el eje HPA también conocido como sistema neuroendocrino, quizás contribuyen sustancialmente a los olvidos característicos de la edad. Incluso, alteraciones en el sistema inmunitario, pueden ser consecuencia de niveles elevados de GC.

A consecuencia de lo anterior cada vez más estudios se centran en analizar el potencial que tienen durante el envejecimiento intervenciones conductuales como la estimulación cognitiva o el ejercicio aeróbico debido a los beneficios que aportan no solo sobre la salud física, sino también a nivel cognitivo o incluso, sobre el estado de ánimo. Los efectos específicos de estas intervenciones a nivel cerebral se han ido conociendo gracias a los trabajos con modelos animales, principalmente roedores, sometidos a programas de enriquecimiento ambiental (EAM), o bien de ejercicio aeróbico (EJ). El término EAM se refiere a una mejora de las condiciones de estabulación de los animales de laboratorio en comparación con las empleadas de forma estándar (Baumans y Van Loo, 2013). Estas condiciones incluyen jaulas de mayor tamaño que contienen objetos y espacios diversos que facilitan el ejercicio, el juego, la exploración, a la vez que permiten a los animales tener un mayor control sobre su ambiente. En algunos paradigmas experimentales, el EAM también puede incluir estimulación social mediante el aumento del número de animales por jaula favoreciendo así, constantes interacciones dinámicas e impredecibles (Stewart y Bayne, 2004). Por su 
parte, el EJ puede tener un carácter forzado o voluntario, dependiendo de si los animales son obligados a correr en ruedas o cintas durante un período concreto del día, o bien si pueden hacerlo de forma voluntaria al disponer de una rueda en su jaula. Estas intervenciones han mostrado afectar al funcionamiento del eje HPA promoviendo un efecto ansiolítico al reducir la cantidad de GC secretados cuando los animales son expuestos a situaciones estresantes y al aumentar la expresión de RG encargados de inhibir su activación (Sampedro-Piquero, Arias, y Begega, 2014). Sin embargo, poco se sabe acerca del impacto que tienen estos programas sobre regiones cerebrales cuyas eferencias, ya sean excitatorias o inhibitorias, controlan la actividad del eje HPA.

De este modo, el objetivo de este trabajo consistió en estudiar la actividad metabólica neuronal, a través de la histoquímica de la citocromo c oxidasa (COx), de diferentes regiones cerebrales implicadas tanto en la activación como en la inhibición del eje HPA, en roedores de 18 meses que fueron expuestos a un programa de EAM o de EJ durante 2 meses. La COx es la enzima terminal o complejo IV del canal de transporte de electrones de la mitocondria y su actividad representa un índice de las demandas energéticas de las neuronas (Méndez-López, Méndez, Sampedro-Piquero, y Arias, 2012; Rubio, Begega, Méndez, Méndez-López, y Arias, 2012; Villarreal, González-Lima, Berndt, y Barea-Rodríguez, 2002).

\section{MÉTODO}

\section{Grupos experimentales}

En este trabajo contamos con un total de 22 ratas Wistar macho (18 meses de edad al inicio del experimento) procedentes del Bioterio de la Universidad de Sevilla. Todos los animales tuvieron acceso a agua y comida ad libitum y fueron mantenidos a una temperatura $\left(20-21^{\circ} \mathrm{C}\right)$, humedad $(65-70 \%)$ y ciclo de luz/oscuridad constante (08:00-20:00h luz / 20:00-08:00 oscuridad). Durante el experimento se cumplió con las directrices 2010/63/UE andRD1201/2005 que hacen referencia a la protección de los animales de experimentación.

Los animales fueron asignados aleatoriamente a tres grupos: grupo control $(\mathrm{CO}, N=6)$, grupo EAM $(N=8)$ y grupo $\mathrm{EJ}(N=8)$.

\section{Enriquecimiento ambiental}

El grupo EAM fue estabulado en una jaula grande $(100 \times 95 \times 54 \mathrm{~cm})$ durante 2 meses, 3h/día (10:00 am/13:00 pm). La jaula contaba con una gran variedad de objetos, como juguetes, plataformas, casas de madera, objetos con olor (naranja, chocolate), sonido (cascabeles, sonajeros) así como, material para la construcción de nido. Además, también se proporcionada a los animales tubos de plástico y cobijos de madera que les permitían cobijarse y les hacían el ambiente más confortable. Estos objetos fueron 
renovados frecuentemente por otros para garantizar la exposición a la novedad así como, niveles elevados de exploración. El resto del día los animales era estabulados en grupos de 4 en jaulas estándar de laboratorio $(55 \times 20 \times 34 \mathrm{~cm})$ sin ningún tipo de objeto estimulante. El grupo CO y EJ fueron estabulados en las jaulas estándar.

Figura 2. Típico ambiente enriquecido en el que se ofrece estimulación motora, sensorial, cognitiva y física a los roedores
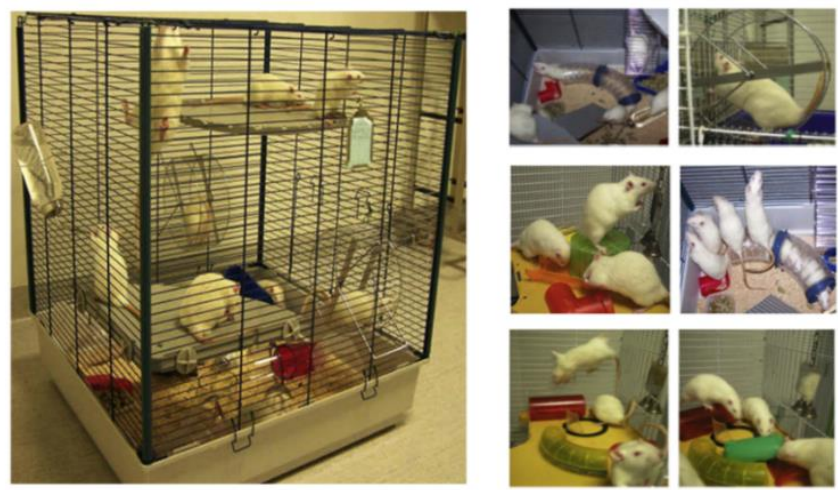

Los objetos son frecuentemente cambiados para motivar las conductas exploratorias en los animales (Fuente: Petrosini et al., 2009).

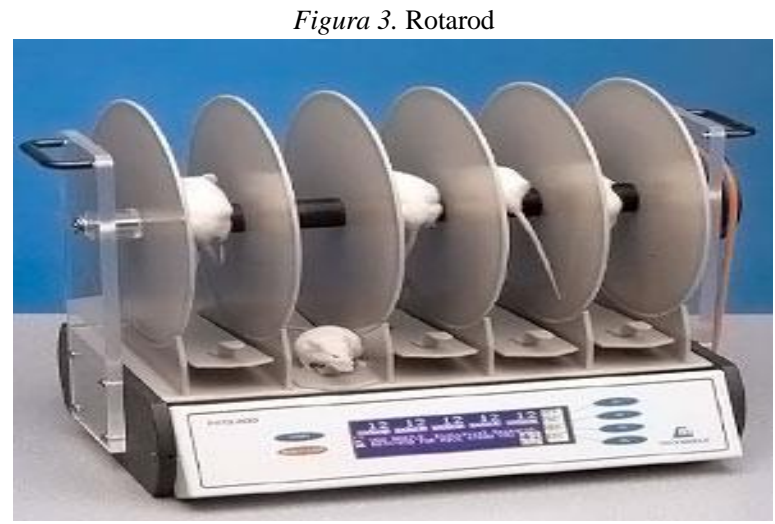

En él los animales son colocados individualmente en cada una de las ruedas disponibles en el sentido contrario del giro de la rueda. A pesar de su carácter forzado, nos permite el control de la frecuencia, duración e intensidad de nuestro protocolo de ejercicio (Fuente: Linton Instrumentation).

\section{Ejercicio aeróbico}

El ejercicio aplicado en nuestro trabajo fue de tipo forzado ya que nos permite controlar con más precisión que tanto el tiempo como la intensidad del protocolo son 
igual para todos los roedores. El ejercicio fue realizado en el aparato Rotarod (47700, Ugo Basile) 3 veces por semana durante 15 minutos a una intensidad de 5 rpm durante un período de 2 meses. Los animales eran colocados en contra del sentido de giro de la rueda y en el caso de caer, eran posicionados de nuevo en ella inmediatamente. La velocidad se mantuvo constante durante todo el experimento.

Figura 4. Representación esquemática de los pasos a seguir en la realización de la histoquímica de la COx

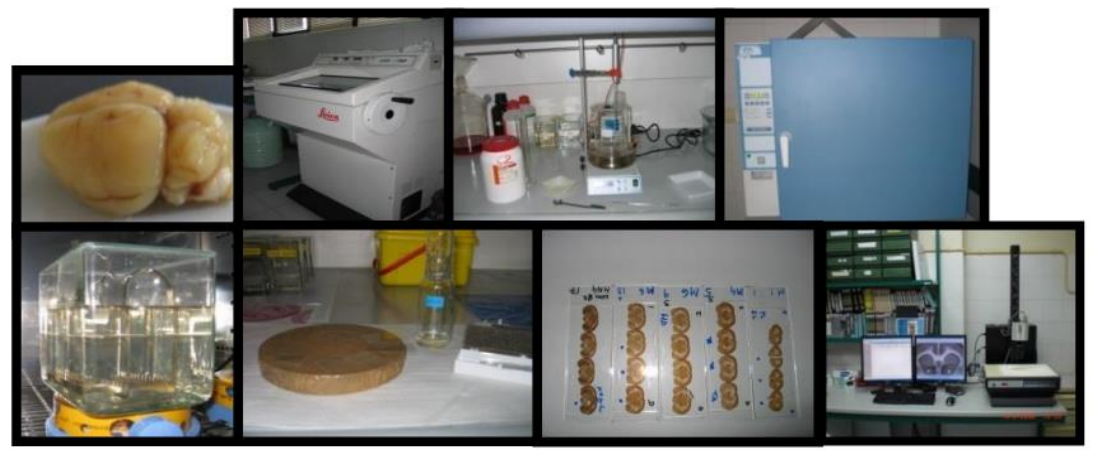

Una vez extraído el cerebro, se sigue con su seriación a $30 \mathrm{~m}$ mediante el criostato. A continuación, se preparan las soluciones necesarias para la tinción y por último, se realiza la cuantificación densitométrica de la COx mediante el programa MCID, InterFocus Imaging Ltd., Linton, England

\section{Histoquímica de la citocromo c oxidasa (COx)}

Los animales fueron sacrificados y sus cerebros congelados en isopentano y almacenados a $-40^{\circ} \mathrm{C}$. Mediante un criostato, a una temperatura de $-20^{\circ} \mathrm{C}$, se obtuvieron secciones de $30 \mu \mathrm{m}$ con las que se realizó la histoquímica de la COx. Para más detalles sobre esta técnica y los productos necesarios para su realización véase Sampedro-Piquero, Zancada-Menéndez, Begega, Cuesta, y Arias, 2013. La cuantificación de la actividad COx se realizó por densitometría óptica con un programa específico para este tipo de análisis (MCID, InterFocus Imaging Ltd., Linton, England). Las regiones de interés en este estudio fueron definidas anatómicamente mediante el atlas de Paxinos y Watson (2005): corteza orbital medial $(4.20 \mathrm{~mm})$, corteza prefrontal (cingulada, prelímbica e infralímbica, $2.70 \mathrm{~mm})$, núcleo accumbens $(1.60 \mathrm{~mm})$, septum lateral y núcleo del lecho de la estría terminal $(-0.26 \mathrm{~mm})$, núcleo paraventricular talámico e hipotalámico $(-1.80 \mathrm{~mm})$, amígdala basolateral y central $(-2.12 \mathrm{~mm})$ e hipocampo dorsal (CA1, CA3, Giro dentado, -3.84 mm) y ventral (CA1, CA3, Giro dentado, $-5.80 \mathrm{~mm}$ ). La figura 4 muestra esquemáticamente el proceso de la histoquímica de la COx.

\section{Análisis estadísticos}

Los datos fueron analizados con el programa SPSS 19.0 (SPSS Inc., Chicago, 
USA) y expresados como media \pm SEM (Tabla 1). Los resultados fueron considerados estadísticamente significativos si $p<0.05$. Con el objetivo de conocer si existían diferencias significativas entre grupos en la actividad metabólica de las regiones cerebrales estudiadas realizamos un análisis multivariado de la varianza (MANOVA). En el caso de que existiesen diferencias significativas entre grupos, el test post hoc de Tukey nos permitió conocer entre qué grupos se daban esas diferencias $(p<0.05)$.

\section{RESULTADOS}

El análisis estadístico MANOVA mostró que existían diferencias significativas entre grupos en la actividad COx de algunas de las regiones analizadas en este trabajo (Corteza cingulada, $F_{2,22}=5.734, p=0.001$; Corteza infralímbica, $F_{2,22}=8.150$, $p=0.003$ ); Núcleo del lecho de la estría terminal, $F_{2,22}=6.040, p=0.000$; Amígdala basolateral, $F_{2,22}=12.505, p=0.001$; Núcleo paraventricular hipotalámico, $F_{2,22}=5.776$, $p=0.011$; CA1 dorsal, $F_{2,22}=9.172, p=0.002$; CA3 dorsal, $F_{2,22}=9.664, p=0.001$; Giro dentado dorsal, $F_{2,22}=6.412, p=0.007$ ).

Tabla 1. Resultados del análisis post hoc

\begin{tabular}{lcc}
\hline & CO & EAM \\
\hline Corteza infralímbica & $22.90 \pm 1.35$ & $19.05 \pm 1.8^{*}$ \\
\hline Amígdala basolateral & $31.53 \pm 5.43$ & $22.04 \pm 2.06^{* *}$ \\
\hline Núcleo paraventricular hipotalámico & $18.72 \pm 1.49$ & $14.92 \pm 1.65^{*}$ \\
\hline Corteza cingulada & CO & EJ \\
\hline Núcleo del lecho de la estría terminal & $22.39 \pm 1.05$ & $26.27 \pm 2.02^{*}$ \\
\hline CA1 dorsal & $18.09 \pm 1.23$ & $22.17 \pm 2.64 *$ \\
\hline CA3 dorsal & $16.76 \pm 1.81$ & $22.59 \pm 1.50^{* *}$ \\
\hline GD dorsal & $14.31 \pm 1.27$ & $21.01 \pm 3.84^{* *}$ \\
\hline$* * p<0.01 ; * p<0.05$ & $27.97 \pm 1.27$ & $30.40 \pm 1.07 *$ \\
\hline
\end{tabular}

\section{DISCUSIÓN}

Nuestros resultados parecen sugerir un efecto beneficioso del EAM y del EJ sobre la hiperactivación del eje HPA descrita durante el envejecimiento, al observar que estas intervenciones son capaces de modular de forma diferente regiones cerebrales que envían proyecciones excitatorias e inhibitorias al núcleo paraventricular hipotalámico, punto de inicio del eje. Así, en el caso del EAM parece que produjo una inhibición de algunas regiones cerebrales, como la corteza infralímbica, la amígdala basolateral o el núcleo paraventricular hipotalámico, encargadas de activar al eje de estrés. En cambio, el EJ parece que redujo la actividad del sistema neuroendocrino mediante la activación de 
regiones cerebrales que lo inhiben, como la corteza cingulada, el núcleo del lecho de la estría terminal y el hipocampo dorsal.

En relación al EAM, varios estudios han mostrado que produce un efecto ansiolítico sobre todo cuando los roedores viejos son expuestos a estresores psicogénicos, como es el caso de un ambiente o situación nueva e impredecible (Fox y cols., 2006). Esto sigue la línea de lo observado en el caso de las personas mayores, en las que con el paso de los años se reduce la conducta social, el comienzo de nuevos aprendizajes y en general, la búsqueda de novedad, quizás debido al estrés que generan estas situaciones (File y Seth, 2003). El efecto positivo del EAM es fácilmente comprensible si tenemos en cuenta que la exposición a la novedad es una de sus características inherentes (Van de Weerd et al., 2002). El cambio constante de los estímulos es considerado un mecanismo de inoculación de estrés, puesto que la repetida introducción de objetos nuevos es comparable a la exposición frecuente a estresores de carácter leve (Larsson, Winblad, y Mohammed, 2002). Sin embargo, otros autores sugieren que el EAM es capaz de reducir los niveles de ansiedad al aumentar el sentido de control que los animales tienen sobre el ambiente (Roy, Belzung, Delarue, y Chapillon, 2001).

Los trabajos a nivel cerebral muestran como esta intervención es capaz de reducir los niveles de hormonas implicadas en la activación del eje HPA, como las hormonas adenocorticotropina, secretada por el núcleo paraventricular del hipotálamo, y los GC, secretados por la corteza de las glándulas adrenales, cuando los animales son expuestos a diferentes estresores (Garrido, 2011). También, el EAM aumenta los niveles de RG en el hipocampo, lo cual facilita la inhibición de la actividad del eje HPA y un restablecimiento de los niveles basales de GC (Olsson, Mohammed, Donaldson, Henriksson, y Seckl, 1994). Por último, el aumento que el EAM provoca sobre la expresión del factor de crecimiento BDNF en el cerebro envejecido, se ha relacionado con una mayor capacidad de resiliencia en estos animales (Segovia, Del Arco, Garrido, de Blas, y Mora, 2008).

En cuanto al EJ aeróbico, varios son los trabajos que también han mostrado un efecto positivo de esta intervención sobre la cognición así como, sobre aspectos emocionales (Pietrelli, López-Costa, Goñi, Brusco, y Basso, 2012). Diferentes autores (Campbell, Rakhshani, Fediuc, Bruni, y Riddell, 2009) mostraron que el ejercicio provoca en un principio un aumento de activación del eje HPA y por tanto, de los niveles de GC en sangre. Estos niveles son gradualmente reducidos a valores normales cuando el ejercicio se prolonga durante un período largo de tiempo promoviendo una respuesta adaptativa ante futuros estresores. Este efecto se relaciona con el fenómeno de supercompensación descrito por Folbrot en 1941 y más tarde discutido por Selye que se refirió a él como síndrome de adaptación general, según el cual una adaptación al trabajo y al proceso de entrenamiento da lugar a adaptaciones homeostáticas ante 
situaciones de mayor demanda. A nivel cerebral, el ejercicio aeróbico provoca, incluso en edades avanzadas, un aumento de la proliferación neuronal, la formación de espinas y ramificaciones dendríticas, la expresión de factores de crecimiento, o la formación de nuevos vasos sanguíneos a partir de los existentes (Stranahan, Lee, y Mattson, 2008). En el caso de las nuevas neuronas, muchos las han relacionado no sólo con procesos de aprendizaje y memoria dependientes del hipocampo, sino también con una mejor capacidad de afrontamiento de situaciones estresantes debido a su efecto ansiolítico (Levone, Cryan, y O’Leary, 2015).

Por tanto, este trabajo parece sugerir que los efectos ansiolíticos de estas intervenciones durante el envejecimiento pueden ser explicados en parte por la modulación que ejercen sobre distintas regiones cerebrales que actúan sobre el eje de estrés. De este modo, y debido a la estrecha relación que existe entre el ámbito cognitivo y el emocional, puede que promover este tipo de actividades en edades avanzadas provoque una mejora de funciones tan importantes para el día a día como la memoria episódica o las funciones ejecutivas. No obstante, se trata de un estudio preliminar en modelo animal por lo que debe de ser contrastado en personas mayores.

\section{CONCLUSIONES}

La estimulación mental y el ejercicio aeróbico son intervenciones conductuales que han mostrado tener grandes beneficios en el ámbito cognitivo en edades avanzadas. Sin embargo, poco es sabido sobre su impacto en el área emocional. En este trabajo, mediante un análisis de la actividad metabólica neuronal, quisimos analizar el grado de activación de regiones cerebrales relacionadas con el eje de estrés en roedores viejos que fueron sometidos a un programa de EAM y de EJ aeróbico durante 2 meses en comparación con otro grupo mantenido en condiciones estándar de laboratorio. Los resultados parecen sugerir que estas intervenciones promueven un efecto ansiolítico quizás mediado en parte por una modulación diferencial del eje HPA, es decir, el EAM inhibe la activación de regiones que lo activan y el EJ aeróbico activa a regiones que lo inhiben. Como línea futura sería interesante complementar estos datos con un análisis de los niveles de GC en sangre o la expresión de GR.

\section{Agradecimientos}

Nos gustaría dar las gracias al estabulario de Sevilla por proporcionarnos la muestra de ratas Wistar macho viejas. También a las técnico Piedad Burgos y Begoña Díaz por su ayuda en el procesamiento del tejido cerebral. Este trabajo fue realizado gracias al contrato de Formación de profesorado universitario (FPU, AP2010-1654) y al proyecto PSI 2013 42704P (MINECO). 


\section{REFERENCIAS}

Baumans, V., y Van Loo, P.L. (2013). How to improve housing condition of laboratory animals: The possibilities of environmental enrichment. Veterinary Journal, 195, 24-32.

Bishop, N., Lu, T., y Yankner, B. (2010). Neural mechanisms of ageing and cognitive decline. Nature, 464, 529-535.

Bizon, J.L., Helm, K.A., Han, J.S., Chun, H.J., Pucilowska, J., Lund, P.K., y Gallagher, M. (2001). Hypothalamic-pituitary-adrenal axis function and corticosterone receptor expression in behaviourally characterized young and aged Long-Evans rats. European Journal of Neuroscience, 14, 1739-1751.

Campbell, J.E., Rakhshani, N., Fediuc, S., Bruni, S., y Riddell, M.C. (2009). Voluntary wheel running initially increases adrenal sensitivity to adrenocorticotrophic hormone, which is attenuated with long-term training. Journal of Applied Physiology, 106, 66-72.

Christensen, K., Doblhammer, G., Rau, R., y Vaupel, J.W. (2009). Ageing populations: the challenges ahead. The Lancet, 374, 1196-1208.

Deary, I.J., Corley, J., Gow, A.J., Harris, S.E., Houlihan, L.M., Marioni, R.E., Penke, L., Rafnsson, S.B., y Starr, J.M. (2009). Age-associated cognitive decline. British Medical Bulletin, 92, 135-152.

Eichenbaum, H., Yonelinas, A.P., y Ranganath, C. (2007). The medial temporal lobe and recognition memory. Annual Review of Neuroscience, 30, 123-152.

File, S.E., y Seth, P. (2003). A review of 25 years of the social interaction test. European Journal of Pharmacology, 463, 35-53.

Fjell, A.M., McEvoy, L., Holland, D., Dale, A.M., y Walhovd, K.B. (2014). What is normal in normal aging? Effects of aging, amyloid and Alzheimer's disease on the cerebral cortex and the hippocampus. Progress in Neurobiology, 117, 20-40.

Fox, C., Merali, Z., y Harrison, C. (2006). Therapeutic and protective effect of environmental enrichment against psychogenic and neurogenic stress. Behavioral Brain Research, 175, $1-8$.

Garrido, P. (2011). Aging and stress: past hypothesis, present approaches and perspectives. Aging and Disease, 2, 80-99.

Jöels, M., Karst, H., De Rijk, R., y de Kloet, E.R. (2008). The coming out of the brain mineralocorticoid receptor. Trends in Neuroscience, 31, 1-7.

Larsson, F., Winblad, B., y Mohammed, A.H. (2002). Psychological stress and environmental adaptation in enriched vs. impoverished housed rats. Pharmacology Biochemistry Behavior, 73, 193-207.

Levone, B.R., Cryan, J.F., y O'Leary, O.F. (2015). Role of adult hipocampal neurogenesis in stress resilience. Neurobiology of Stress, 1, 147-155.

Méndez-López, M., Méndez, M., Sampedro-Piquero, P., y Arias, J.L. (2012). Spatial learningrelated changes in metabolic activity of limbic structures at different posttasks delays. Journal of Neuroscience Research, 91, 151-159.

O'Callaghan, R.M., Griffin, E.W., y Kelly, A.M. (2009). Long-term treadmill exposure protects against age-related neurodegenerative change in the rat hippocampus. Hippocampus, 19, 1019-1029.

Olsson, T., Mohammed, A.H., Donaldson, L.F., Henriksson, B.G., y Seckl, J.R. (1994). Glucocorticoid receptor and NGFI-A gene expression are induced in the hippocampus after environmental enrichment in adult rats. Molecular Brain Research, 23, 349-353.

Organización mundial de la salud (OMS) y Alzheimer's disease international (2013). Demencia: una prioridad de salud pública. (c) Organización Mundial de la Salud, 2013. 
Paxinos, G., y Watson, C. (2005). The Rat Brain in Stereotaxic Coordinates the New Coronal Set ( 5 th ed.). London: Elsevier Academic Press.

Petersen, R.C. (2011). Mild cognitive impairment. The New England Journal of Medicine, 364, 2227-2234.

Petrosini, L., De Bartolo, P., Foti, F., Gelfo, F., Cutuli, D., Leggio, M.G., y Mandolesi, L. (2009). On whether the environmental enrichment may provide cognitive and brain reserves. Brain Research Reviews, 61, 221-239.

Pietrelli, A., López-Costa, J., Goñi, R., Brusco, A., y Basso, N. (2012). Aerobic exercise prevents age-dependent cognitive decline and reduces anxiety-related behaviors in middle-aged and old rats. Neuroscience, 202, 252-266.

Plassman, B.L., Langa, K.M., Fisher, G.G., Heeringa, S.G., Weir, D.R., Ofstedal, M.B., Burke, J.R., Hurd, M.D., Potter, G.G., Rodgers, W.L., Steffens, D.C., McArdle, J.J., Willis, R.J., y Wallace, R.B. (2008). Prevalence of cognitive impairment without dementia in the United States. Annal of Internal Medicine, 148, 427-434.

Prenderville, J., Kennedy, P., Dinan, T., y Cryan, J. (2014). Adding fuel to the fire: the impact of stress on the ageing brain. Trends in Neuroscience, 38, 13-25.

Prickaerts, J., y Steckler, T. (2005). Effects of glucocorticoids on emotion and cognitive processes in animals. Techniques in the Behavioral and Neural Sciences, 15, 359-385.

Roy, V., Belzung, C., Delarue, C., y Chapillon, P. (2001). Environmental enrichment in BALB/c mice: effects in classical tests of anxiety and exposure to a predatory odor. Physiology $y$ Behavior, 74, 313-320.

Rubio, S., Begega, A., Méndez, M., Méndez-López, M., y Arias, J.L. (2012). Similarities and differences between the brain networks underlying allocentric and egocentric spatial learning in rat revealed by cytochrome c oxidase. Neuroscience, 223, 174-182.

Sampedro-Piquero, P., Arias, J.L., y Begega, A. (2014). Behavioural testing-related changes in the expression of Synapsin I and glucocorticoid receptors in standard and enriched aged Wistar rats. Experimental Gerontology, 58, 292-302.

Sampedro-Piquero, P., Begega, A., Zancada-Menéndez, C., Cuesta, M., y Arias, J.L. (2013). Agedependent effects of environmental enrichment and spatial memory in Wistar rats. Neuroscience, 248, 43-53.

Sandi, C., y Pinelo-Nava, M.T. (2007). Stress and memory: Behavioral effects and neurobiological mechanisms. Neural Plasticity, 2007, 1-20.

Segovia, G., Del Arco, A., Garrido, P., de Blas, M., y Mora, F. (2008). Environmental enrichment reduces the response to stress of the cholinergic system in the prefrontal cortex during aging. Neurochemistry International, 52, 1198-1203.

Stewart, K.L., y Bayne, K. (2004). Environmental enrichment for laboratory animals. In: Reuter, J.D., Suckow, M.A. (Eds.), Laboratory Animal Medicine and Management, International veterinary information service, B2520.0404.

Stranahan, A.M., Lee, K., y Mattson, M.P. (2008). Central mechanisms of HPA axis regulation by voluntary exercise. Neuromolecular Medicine, 10, 118-127.

Tasker, J.G., y Herman, J.P. (2011). Mechanisms of rapid glucocorticoid feedback inhibition of the hypothalamic-pituitary-adrenal axis. Stress, 14, 398-406.

Van de Weerd, H.A., Aarsen, E.L., Mulder, A., Kruitwagen, C.L., Hendriksen, C.F., y Baumans, V. (2002). Effects of environmental enrichment for mice: Variation in experimental results. Journal of Applied Animal Welfare Science, 5, 87-109.

Villarreal, J.S., González-Lima, F., Berndt, J., y Barea-Rodríguez, E.J. (2002). Water maze training in aged rats: Effects on brain metabolic capacity and behavior. Brain Research, 939, 43-51. 
Wolitzkty-Taylor, K.B., Castriotta, N., Lenze, E.J., Stanley, M.A., y Craske, M.G. (2010). Anxiety disorders in older adults: a comprehensive review. Depression and anxiety, 27, 190-211.

Recibido: 26 de abril de 2015 Recepción Modificaciones: 31 de julio de 2015 Aceptado: 4 de agosto de 2015 